\title{
A SURVEY ON PELVIC BONE FRACTURE DETECTION
}

\author{
Ligisha $\mathbf{P}^{1}, \mathbf{S}$ Bhavani ${ }^{2}$ \\ ${ }^{1}$ Department of ECE, Karpagam University \\ ${ }^{2} \mathrm{HOD} \&$ Associate Professor, Department of ECE, Karpagam University
}

\begin{abstract}
Traumatic injury is common and potentially devastating with pelvic fractures being a major cause of patient mortality. Detection of pelvic injuries are challenging due to the visual characteristic and complexity in fracture over that region.. The paper deals with the comparison of a few methods used for pelvic bone fracture detection. The survey is based on different modalities like X ray, CT and MRI. The methods have been documented in a way that aids ease of interpretation. The study will help in designing a more powerful automatic pelvic bone fracture detection method.
\end{abstract}

Keywords -Image Processing, Pelvic Fracture, Graph Cut, Mean, Gaussian Curvatures, ANN, Deformable Model.

\section{INTRODUCTION}

In modern medicine medical imaging have undergone major advancements. Medical imaging seeks to reveal internal structures hidden by the skin as well as to diagnose and treat disease. Magnetic Resonance Imaging, Ultra Sound, Computed Tomography, Nuclear Imaging, including Single Photo Emission Computed Tomography and Photo Emission Tomography are some diagnostic medical imaging tools. Quick and accurate diagnosis can be crucial to the success of any prescribed treatment.

It is very difficult to identify a fracture in the pelvic structure due to the complexity of the pelvic structure and the visual characteristics by location. The purpose of this paper is to investigate the methods used in the detection of fractures in pelvic region. Pelvic fractures resulting from trauma cause great morbidity and mortality, as they may lead to laceration of the surrounding soft tissue, neural and vascular structures and nearby organs.

\section{METHOD}

Only a few methods are proposed for the detection of pelvic bone fracture. A comparison of these methods is carried out in this paper. This will help the reader to understand the amount of research carried out in this field.

\subsection{Standard ASM}

Standard ASM uses standard deformable Active Shape Models. A statistical shape and intensity level model of a desired material is obtained by using training images. The training images will be labeled with landmark points. The object in a new image is located by iteratively deforming the shape model. Pixels are sampled along the profile normal to its current position and the best new position of each landmark is determined by a search iteration procedure. At the end of each iteration, updating of the shape parameters are done so as to fit the new land mark position
The boundary determined by ASM is separated in to two windows and the fracture in each window is detected separately. The image contrast is then increased and then two stages 2D discrete wavelet transforms are applied. The first stage of the $2 \mathrm{D}$ discrete waveform removes noise from the image. The DWT, extracts the horizontal, vertical and diagonal detail coefficients. The application of the DWT highlights the bone boundary. The traced bone boundary is converted into binary format. Morphological operations such as opening and thinning of the image are performed. The boundaries of the ring are traced from the resulting binary image. If the boundary is single and uninterrupted then there is no fracture in the image. If the boundary is multiple then it shows the presence of fracture in the image

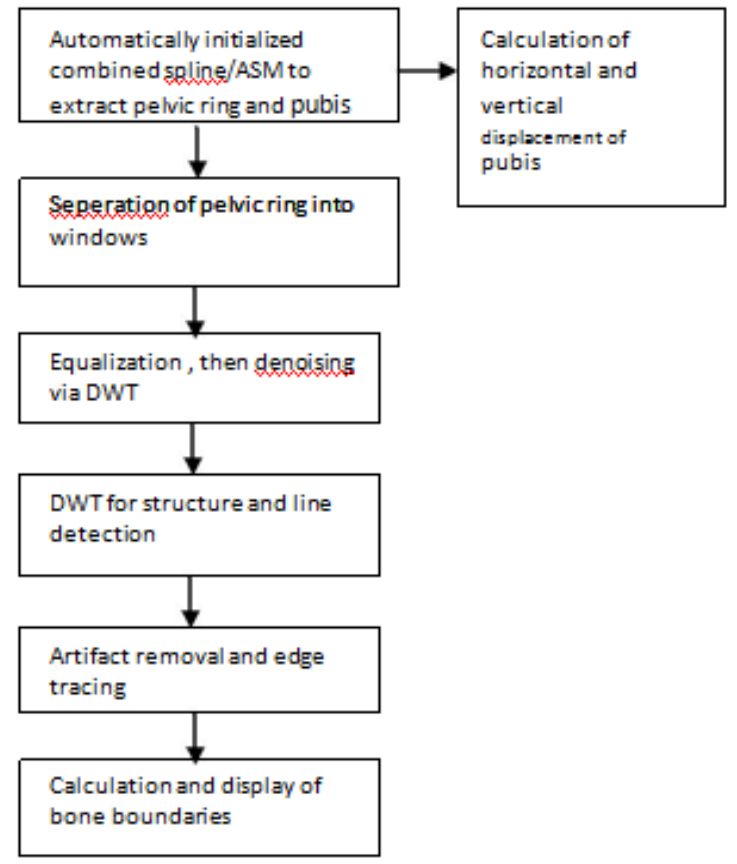

Fig. 1: Fracture detection using ASM 
The main drawback of this system is that the system is highly sensitive to initialization. The system is semiautomatic, the image have to be placed manually. If there is any error in the initial position of the system then it may cause error in the detection process.

\subsection{Registered ASM}

Instead of X ray images, CT images are considered for fracture detection in Registered Active Shape Model. RASM overcomes the disadvantage of ASM. In RASM the image is first registered to remove the initialization error. Preprocessing steps are performed to extract the region of interest. Then morphological operations such as image enhancement is done to increase the brightness of the bone region and to suppress the unwanted regions. After that the edges are detected using canny edge detection. After edge detection the segmentation process is completed using registered ASM. The process is similar to that done in the standard ASM but here an initialization process is followed. The training models are accurately and automatically placed. This reduces the initialization error and manual interference.

Segmentation using RASM is followed by a number of steps for fracture detection. The first step is to separate the image into two. The two regions are used to obtain a set of multiple adaptive windows..Multiple boundaries on the selected windows shows the presence of fracture in the image. The severity of the fracture can be determined from the number of multiple boundaries.

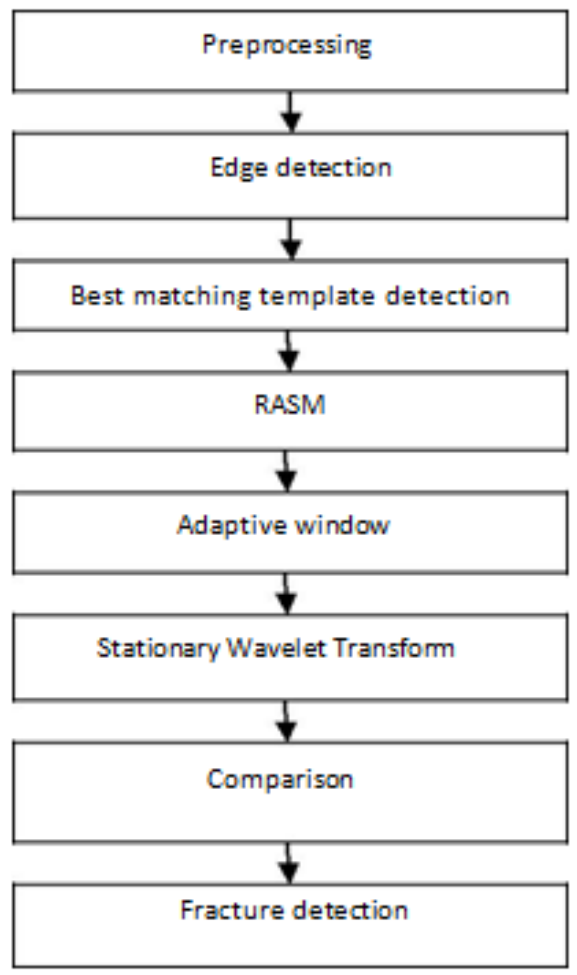

Fig. 2: Fracture detection using RASM
The system overcomes the disadvantage of standard ASM which is highly sensitive to initialization. Any way lot of training images are required for the process and also it is time consuming. In this system about $7.8 \%$ failure were reported.

\subsection{Graph Cuts and Curvatures}

A combination of graph theory and differential geometry is used to detect the fracture in CT images. The first method follows graph theory for fracture detection. It is a semiautomatic approach. In this approach a bounding box surrounding the fracture is input by the user. The pixels inside the bounding box is potentially segmented into two classes, namely - object or bony structure with high intensities and -background or the fracture plus soft tissues with low intensities. From the object and the background two special terminal nodes are formed. The special terminal nodes are denoted as the source and the sink. All the pixels in the source node and the sink node are connected using links. A flow network is formed. Cut vertices of the background shows the fracture points. In the second method differential geometry is utilized. The Gaussian curvature and the mean are used for fracture detection. These parameters can be calculated from the principal curvatures.

Fracture detection is done from a joint decision and neighborhood analysis. A pixel is considered to be a fracture if it is a cut point and it is a valley surface. After joint decision post processing is performed. A linear pattern is formed the fracture along with its neighboring bony pixels. To obtain the orientation of this linear pattern a circular neighborhood of is considered, centering each candidate fracture point in the segmented output from the graph cut. A candidate fracture point is considered to be a true fracture point if any two pixels on the circumference of the above circular neighborhood subtending angles $\theta$ and $\theta+\pi$ with respect to $\mathrm{x}_{1}$ axis belong to the object or class.

\subsection{Coarse to Fine Strategy}

In this method two steps are followed for fracture detection. In the first step coarse fracture localization is done. Intensity and curvature profile measurements are used to obtain the coarse fracture localization. A fracture means a low intensity surrounded by a number of high intensities. The volume with fracture will be having a high average intensity. In addition to this the values of mean and Gaussian curvature is also used to determine the coarse localization. After this step the approximate region of fracture is located. In the second step precise fracture detection is done by using two parallel methods. The methods used are $3 \mathrm{D}$ graph cuts and spatially consistent valleys.. 


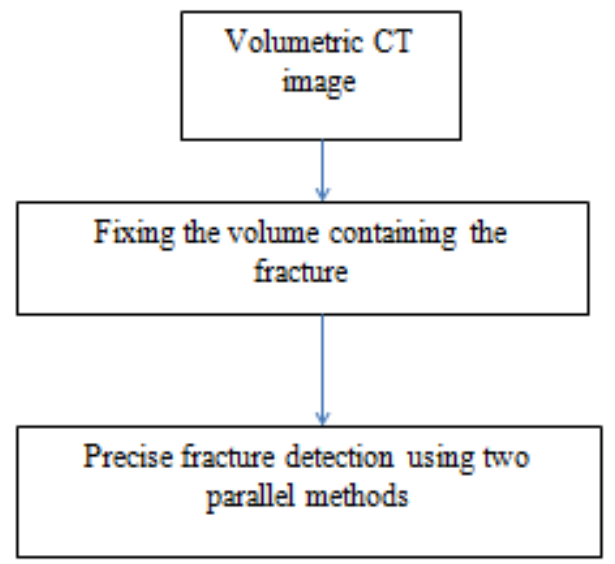

Fig. 3: Fracture detection using a coarse to fine strategy

Precise fracture detection utilizes the two methods in parallel as discussed in the case of graph cut and curvature method. In the first method 3D graph cuts are used. All the valleys in each slice are detected. From the object and the background two special terminal nodes are formed. The special terminal nodes are denoted as the source and the sink. All the pixels in the source node and the sink node are connected using links. A flow network is formed. Cut vertices of the background shows the fracture points. The minimum cut in this method is identified by utilizing special algorithm known as FordFulkerson algorithm. In the second method spatially consistent valleys are used for fracture detection.

All the valleys in the $2 \mathrm{D}$ slice are detected. A valley detected in several slices is considered as fracture.

A joint decision in fracture detection is made based on $3 \mathrm{D}$ graph cut method and spatially consistent valley method. A fracture is identified if there is a cut point in the 3D graph and it is a spatially consistent valley. Accurate detection can be performed by using this joint decision as false positives can be easily removed.

\subsection{GLCM}

Fracture is detected from X ray images using Grey Level Co occurrence Matrix. The first step in this method is image preprocessing. The image is converted in to binary. This is to separate the bone region from the soft tissue region. The soft tissue region can be considered to be noise in this image processing because here the area of interest is the bone region. Further processing of the image is done by using its gray scale histogram. The grey scale histogram carries only intensity information. The values of red, green and blue primaries are necessary to convert an image into its gray scale histogram.

$$
\mathrm{C}_{\text {linear }}=\mathrm{C}_{\mathrm{srgb}} / 12.92 \mathrm{for} \quad \mathrm{Csrgb} \leq 0.04045
$$

$\&$

$$
\mathrm{C}_{\text {linear }}=\left[\left(\mathrm{C}_{\text {srgb }}+0.055\right) / 1.055\right]^{2.4} \text { for } \quad \mathrm{Csrgb} \leq 0.04045
$$

Where the $\mathrm{C}_{\text {srgb }}$ is any of the three gamma compressed

RGB primaries in the range $[0,1]$ and $C_{\text {linear }}$ is the corresponding linear intensity value. Then luminance is calculated as a weighted sum of the three linear intensity values.

The Gray Level Co-occurrence Matrix is a statistical method of examining texture that considers the spatial relationship of pixels. The GLCM functions characterize the texture of an image by calculating how often pairs of pixel with specific values and in a specified spatial relationship occur in an image. After creating GLCM several statistics can be derived. These statistics provide information about the texture of an image. Contrast measures the local variations in the Gray Level Co-occurrence Matrix. Correlation measures the joint probability occurrence of the specified pixel pairs. Energy provides the sum of the squared elements in the GLCM. Homogeneity measures the closeness of the distribution of elements in the GLCM to the GLCM diagonal.. A number of texture features may be extracted from the GLCM. After obtaining the mean and the variance of GLCM, four statistical values which are contrast, correlation, energy homogeneity are analyzed. The algorithm compares the values and classifies the fractured and non-fractured pelvic structures. The accuracy of this system is $86.67 \%$. The disadvantage of the system is that the system indicates the area of the fracture, not the exact image of the fracture. More over the type of the fracture cannot be identified by this method.

\section{CONCLUSION}

The pelvic bone fracture detection methods discussed above are successful and promising. But each of these methods have disadvantages as stated in each section. Theuse of ANNs for medical image segmentationsimplifies the process when dealing with complex images. The most used ANN for segmentation in medical imaging is the Feed Forward Neural Network. The Feed Forward Network is less noisy. So a combination of a Feed Forward Neural Network with some deformable model can attain maximum accuracy for pelvic bone fracture detection.

\section{REFERENCES}

[1]. R. Smith and K.Najarian - Automated Segmentation of Pelvic bone structure in $\mathrm{x}$ ray radiographs using active shape models and directed Hough transforms in the IEEE International conference on bioinformatics and biomedicine workshop.IEEE,2008,pp.56-63

[2]. L.C. Chien. H.M. Cheng. W.C.Cheng and M.C. TsaiFracture and Risk Factors for Mortality. A Population-Based study in Taiwan, EurJ Trauma Emerg Surg. 36:131-137, 2010 [3]. D. Demetriades, M.Karaiskakis, K. Alo, Velmahos, and L. Chan, - Pelvic Fractures : Epidemiology and Predictors of Associated Abdominal Injuries and Outcomes, Am CollSurg, 195: 1-10, 2002. 
[4]. J, Wu, P. Davuluri, K. Ward C. Cockrell, R. Hobson, K. Najarian, --A New Hierarchical method for multilevel Segmentation of bone in pelvic C T scans, I 33 Annual International Conference of the IEEE Engineering in Medicine And Biology Society (EMBC 11), pp.33 93 402,Aug 2011

[5]. S. J. Slater, and D. A. Barron, -- Pelvic fractures-A guide to classification and management, European Journal Radiology, 74 (1) :16-23, 2010.

[6]. M. R. Grotz, M. K. Allami, P. Harood, H. C. Pape, C. Krettek, and P. V. Giannoduis, -- Open pelvic fractures : Epidemiology, current concepts of management and outcome, Injury, 36 (1): 1-13 2005. S. Vasilache and K. Najarian, -automated Bone Segmentation from Pelvic C T images, Proc.IEEE Wkshp. Bioinformatics and Biomedicine (BIBMW), Philadelphia, PA , 41-47,2008.

[7]. R. A. Zoroofi, Y.Sato T. Sasama, T Nishi, N. Sugano, K. Yonenobu, H. Yoshikwa, T. Ochi, and S. Tamura, -Automated Segmentation of Acetabulum and Femural Head Tech. in Biomedicine, 7(4); 329-343, 2003

[8]. J. Fornaro, G. Szekely, and M. Harders, Semiautomatic Segmentation of Fractured Pelvic Bones for Surgicalplanning Proc. Int. Symp. O Biomedical Simulation (ISBMS), springer LNCS 5958; 82-89, 2010.

[9]. J. Wu, P. Davuluri, K. Ward, C. Cockrell, R. Hobson, and K.Nagarian,--Fracture Dettection in Trauma Pelvic C T images, Int. J. Biomed. Imaging, PP. 1-16, 2011.

[10]. J. Edmonds and R. M. Karp, - Theoretical improvements in algorithmic efficiency for network flow problems, Journel of the ACM, 19(2) 248-264, 1972.

[11]. T. F. Cootes, C. J. Taylor, D. H. Cooper, and J. GrahanActive Shape Models- their training and application, Computer Vision And Image Understanding Vol, No. Ipp.38$59,1995$.

[12]. J. Wu, P. Davuluri, K. Ward, C. Cockrell, R. Hobson, and K. Nagarian, -Fracture Detection in Trauma Pelvic C T images , Int. J. Biomed. Imaging, pp.1 -16, 2011. 